\title{
Recent developments in maser theory
}

\author{
Moshe Elitzur \\ Physics \& Astronomy Dept., University of Kentucky, Lexington, KY 40506-0055, USA \\ email: moshe@pa.uky.edu
}

\begin{abstract}
This review covers selected developments in maser theory since the previous meeting, Cosmic Masers: From Proto-Stars to Black Holes (Migenes \& Reid 2002). Topics included are time variability of fundamental constants, pumping of $\mathrm{OH}$ megamasers and indicators for differentiating disks from bi-directional outflows.
\end{abstract}

Keywords. masers, polarization, radiative transfer, accretion disks, cosmology: miscellaneous

\section{Fun with masers}

Rather then maser theory, the first couple of items covered here can be more properly described as "fun with masers". These items were selected for discussion because they do not belong under the title of any other talk in this meeting. Both involve weak $\mathrm{OH}$ signals that lead to spectacular results.

\subsection{How do we know masers exist?}

There are numerous arguments why the radiation from various astronomical sources involves maser amplification (e.g., Elitzur 1992). But the only direct proof can come from measurements of excitation temperatures that yield negative values. The well known technique for measuring a line excitation temperature, $T_{x}$, requires a molecular cloud in front of a distance continuum source that provides background radiation with measurable brightness temperature $T_{b}$ at the transition frequency. The measured brightness temperatures in the "off" and "on" positions, away from and toward the background source, obey

$$
T^{\mathrm{off}}=T_{x}\left(1-e^{-\tau}\right), \quad T^{\mathrm{on}}=\left(T_{x}-T_{e}\right)\left(1-e^{-\tau}\right)
$$

where $\tau$ is the line optical depth of the cloud. The two measurements yield two equations for the two unknowns $T_{x}$ and $\tau$. In the classic experiment by Rieu et al. (1976), the $\mathrm{OH}$ main lines from a cloud in front of the extragalactic radio source $3 \mathrm{C} 123$ switched from emission in the off-source position to on-source absorption. The excitation temperature of both lines is $T_{x} \simeq 5-7 \mathrm{~K}$. But in the case of the $1720 \mathrm{MHz}$ line, off-source emission turned into even stronger emission in the on-source position, indicating that the cloud amplifies the background radiation with $\tau \simeq-0.1$. The corresponding excitation temperature is $T_{x}(1720) \simeq-10 \mathrm{~K}$.

Almost 30 years later, Weisberg et al. (2005) came up with a beautiful variant of this approach in $\mathrm{OH}$ spectral measurements toward 18 pulsars. One member of the sample, pulsar B1641-45, emits every 0.455 seconds a pulse of radio radiation that passes through an $\mathrm{OH}$ cloud on its way toward Earth. The entire system is positioned in front of an HII region that provides additional, steady background radiation. When the pulsar is off, spectra of the $\mathrm{OH}$ ground-state lines detected from the cloud display absorption features in the 1612, 1665 and $1667 \mathrm{MHz}$ lines and an emission feature at $1720 \mathrm{MHz}$. When the pulsar is on, passage of its radiation through the cloud deepens the absorption features. But at $1720 \mathrm{MHz}$ the emission feature becomes stronger, showing that at this frequency 
the pulsar radiation is amplified by passing through the intervening screen. This is the equivalent of an object amplifying a background light instead of casting a shadow. It is the same effect as observed earlier by Rieu et al., but it does not involve any change in the telescope pointing. The pulsar method also increases confidence in the results, thanks to the repeated detection of amplified signals that arrive more than twice every second. The amplification again is modest, $\tau$ is only $\sim-0.05$. Although the input signals are amplified by only a few percent in both cases, these weak masers are the only unambiguous direct evidence for amplification.

Figure 1. OH satellite line spectra toward PMN J01340931 plotted against barycentric velocity relative to the source redshift, $z=0.76355$. (A) 1720 $\mathrm{MHz}$ transition; (B) $1612 \mathrm{MHz}$ transition; (C) sum of the 1612 and 1720 $\mathrm{MHz}$ spectra; this spectrum is consistent with noise. (Kanekar et al. 2005)

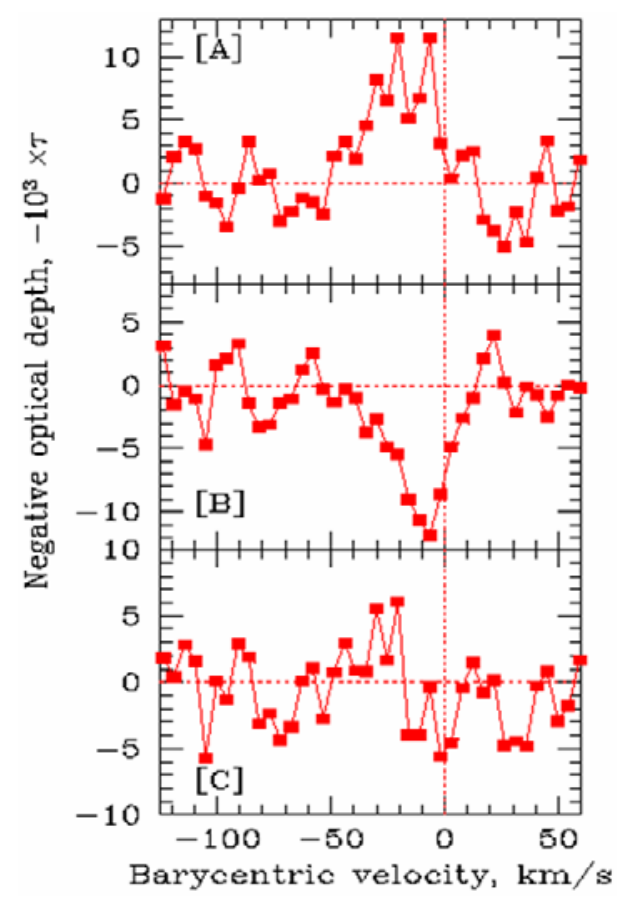

\subsection{Are fundamental constants truly constant?}

A cosmological implication of many variants of string theories is that fundamental constants such as the fine structure constant $\alpha=e^{2} / \hbar c$, the electron-proton mass ratio $m_{\mathrm{e}} / m_{\mathrm{p}}$, etc. should vary with time. Indeed, from measurements of QSO absorption lines it has been suggested that a variation $\Delta \alpha / \alpha \sim 10^{-5}$ has possibly occurred between the $z=3.5$ epoch and the present (Murphy et al. 2001). However, the uncertainty in this suggestion is substantial because measurements based on atomic lines are faced with fundamental difficulties. Comparing the frequencies of different lines of the same atom does not yield information on $\alpha$ because the entire spectrum of a given species has the same $\alpha$-dependence, therefore frequency shifts that would result from a change in $\alpha$ cannot be distinguished from the effects of redshift. And comparison of lines of different atoms suffers from the uncertainty arising from the possibly different locations, and different $z$, of the species.

Darling (2003) pointed out that the complexity of the $\mathrm{OH}$ spectrum makes it most suitable for getting around this problem. The centimeter $\mathrm{OH}$ transitions are a combination of hyperfine splitting and $\Lambda$-doubling, and the two effects depend differently on $\alpha$. As a result, by comparing the frequencies of different $\mathrm{OH}$ radio transitions one can constrain the cosmic evolution of $\alpha$ from a single species, avoiding systematic errors in $\alpha$ 
measurements from multiple species which may have relative velocity offsets. The problem of different locations still exists, though, because different $\mathrm{OH}$ lines may still arise from different locations because of preferential excitations in different environments. This problem was solved by Kanekar et al. (2005) who noted that many weak signals from the $\mathrm{OH}$ ground-state satellite lines appear in conjugate pairs of absorption and emission. The two halves of the $\Lambda$-doublet consist of pairs of hyperfine states. Because of the different overall spins of hyperfine states, their coupling to different excited states makes it easy to transfer population between them without affecting the overall population of each half of the $\Lambda$-doublet. The result is an overpopulation, or even inversion, of one satellite line and a mirror under-population of the other (see, e.g., Elitzur 1992).

Taking $\mathrm{OH}$ spectra from the $z \sim 0.765$ gravitational lens toward PMN J01340931, Kanekar et al. identified a conjugate pair of $1612 \mathrm{MHz}$ absorption and $1720 \mathrm{MHz}$ emission, shown in Figure 1. As is evident from the figure's bottom panel, the sum of the two satellite lines produces a flat spectrum consistent with noise, a strong indication that they originate from the same region. The deduced line frequencies place tight bounds on the variation of fundamental constants over the past $\sim 6.5 \mathrm{G}$ yr: $\Delta \alpha / \alpha<6.7 \times 10^{-6}$ and a similarly strong upper limit of $1.4 \times 10^{-5}$ for $m_{\mathrm{e}} / m_{\mathrm{p}}$. These fundamental constants have not changed, to within this tight tolerance, for half the age of the Universe.

\section{2. $\mathrm{OH}$ megamasers}

$\mathrm{OH}$ megamasers (OHMM) are extremely luminous extragalactic sources with isotropic luminosities a million or more times greater than their Galactic $\mathrm{OH}$ maser counterparts. They are found in the nuclear regions of luminous infrared galaxies where intense star formation is occurring. The "standard model" for OHMM was introduced by Baan (1985), who proposed that the maser emission is produced by low gain unsaturated amplification of background radio continuum. This proposal was confirmed by the Henkel \& Wilson (1990) comprehensive study of OHMM. However, compact maser emission discovered in subsequent VLBI observations produced conflicts with the standard model, conflicts that reached their climax around the time of the previous maser meeting (Lonsdale 2002). Strong compact masers without correspondingly bright continuum background implied amplification factors in excess of 800. In addition, the 1667/1665 line ratios of the compact masers greatly exceeded those of the diffuse OHMM emission. An additional surprise of these observations was that linewidths remained large (tens of $\mathrm{km} \mathrm{s}^{-1}$ ) even on the smallest observed angular scales. This has led to the suggestion that there are two different modes of OHMM operation - low gain, unsaturated amplification responsible for the diffuse maser emission and high gain, saturated emission producing the compact sources. It was also suggested that the two classes may have different pumping mechanisms (Lonsdale 2002).

A major recent development is the realization that a two-component picture is not necessary. The compact masers can be explained within the standard model as chance alignment of low-gain maser clumps. The key was the interferometric observations of IIIZw35 by Pihlström et al. (2001) and the modeling by the same authors and later, in greater detail, by Parra et al. (2005). The model explains both the diffuse and compact emission from IIIZw35 by assuming a clumpy maser medium. Each cloud generates low gain, unsaturated emission, and strong compact emission occurs when the line of sight intersects more than one cloud. A similar model has subsequently been used to explain the megamaser emission from IRAS 17208-0014 (Momjian et al. 2006). The clumpy maser model also explains the observation that compact masers are always found embedded in the diffuse emission and do not occur in isolation (Lonsdale 2002). Thus the only 
additional ingredient necessary to supplement Baan's "standard model" is clumpiness the OHMM phenomenon is explained as the amplification of background radio continuum by low gain $\left(\tau_{1667} \lesssim 1\right)$ clumps.

Lockett \& Elitzur (2007) performed pumping calculations to explain the OHMM inversion process. Pumping models should explain the outstanding properties of OHMM emission: (1) The line ratio of observed fluxes always obeys $F(1667) / F(1665)>1$ and the satellite lines are always weaker than the main lines. By comparison, in evolved stars the main lines emission region displays a similar behavior - $1667 \mathrm{MHz}$ is the strongest maser, and the satellite line emission from that region is weaker than either main line. In contrast, Galactic star forming regions show a different, more complex emission pattern. Generally, the main lines are stronger than the satellite lines and display $F(1667) / F(1665)<1$, the opposite of EHM and evolved stars. But there are sources in which the strongest line is the $1665 \mathrm{MHz}$ is and some where it is a satellite line.
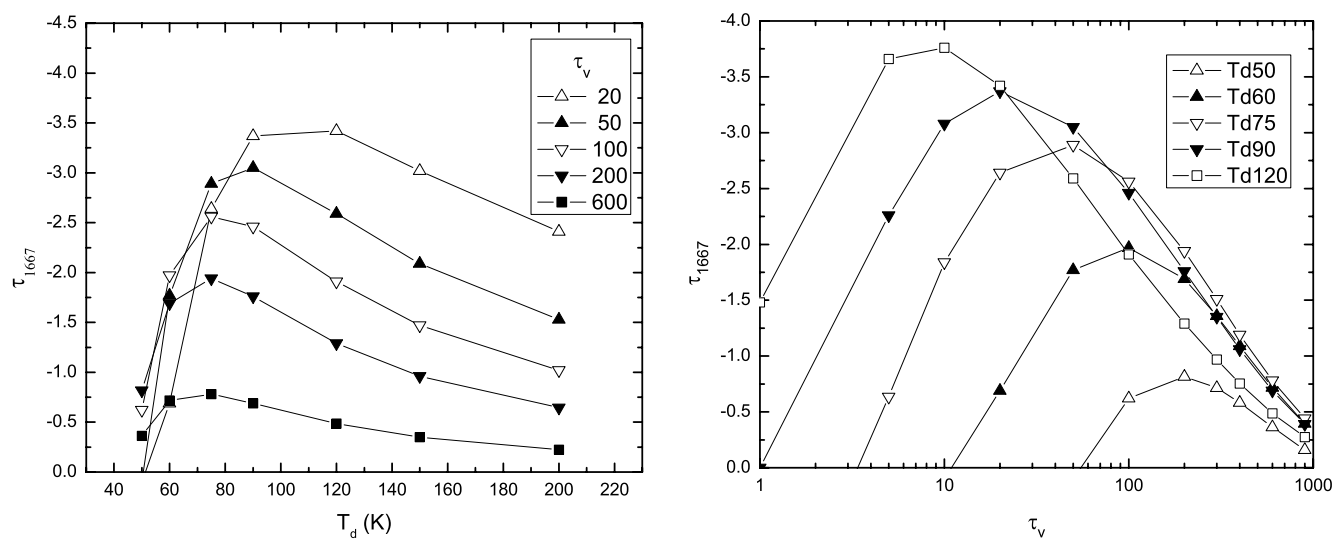

Figure 2. Dependence of the $1667 \mathrm{MHz}$ maser optical depth, $\tau_{1667}$, on the parameters of the IR pumping flux (see eq. 2.1). Left: $\tau_{1667}$ versus dust temperature for varying dust optical depth. Right: $\tau_{1667}$ versus dust opacity for different dust temperatures (Lockett \& Elitzur 2007).

In the Lockett \& Elitzur models, the inversion arises from radiative excitations by far-IR radiation (FIR). The FIR radiation field is described by the simple expression

$$
F_{\nu}=B_{\nu}\left(T_{d}\right)\left(1-e^{-\tau_{\nu}}\right),
$$

corresponding to emission from dust with a uniform temperature $T_{d}$ and optical depth $\tau_{\nu}$. The frequency dependence of $\tau_{\nu}$ follows that of standard interstellar dust so the only free parameters are $T_{d}$ and $\tau_{V}$, the dust optical depth at visual. Combinations of parameters that produce main lines inversion are shown in figure 2. The left panel shows the 1667 $\mathrm{MHz}$ optical depth versus dust temperature for a range of dust opacities. A minimum dust temperature of $\sim 45 \mathrm{~K}$ is needed for maser production. Increasing the dust temperature increases the maser strength up to a point, after which it decreases. Maximum maser inversion occurs for dust temperatures between $\sim 70-120 \mathrm{~K}$. The right panel shows the $1667 \mathrm{MHz}$ optical depth versus dust opacity for a range of dust temperatures. Two important results can be seen in this figure. First, when the opacity becomes large the dust radiation approaches that of a black-body and the maser begins to thermalize. At $\tau_{\mathrm{V}} \gtrsim 200$ there is little dependence of maser strength on dust temperature. Second, for each dust temperature there is a minimum dust opacity needed for inversion. This implies that a minimum FIR pumping flux is needed for maser production. Further understanding can be found by examining the maser dependence on the FIR intensity 


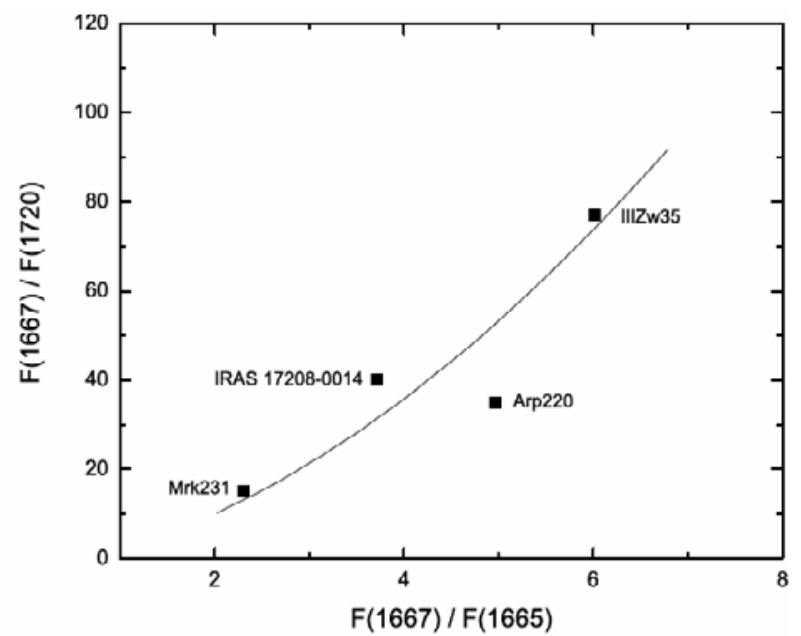

Figure 3. "Color-color" diagram of $\mathrm{OH}$ maser line ratios. The line shows the variation when all four lines have the same excitation temperature, which is the situation when the linewidth exceeds $\sim 10 \mathrm{~km} \mathrm{~s}^{-1}$, as $\tau_{1667}$ increases from 0.5 at the curve's lower end to 4 at its upper end. Data are from Baan et al. 1989, 1992 and Martin et al 1989 (Lockett \& Elitzur 2007).

in the maser pump line at $53 \mu \mathrm{m}$. The pumping flux is determined by the product of the two factors in equation 2.1. The first is the Planck function, which depends on dust temperature, the second is the self-absorption term, which depends on the dust opacity at the pumping frequency. The FIR flux is increased by increasing either the dust temperature or its opacity. As a result, a larger flux is needed for a lower dust temperature, indicating that the FIR spectral shape is important for maser pumping. Thus a smaller IR luminosity can support inversion as long as the dust temperature is sufficiently high — in agreement with observations (Baan 1989, Darling \& Giovanelli 2002). Similarly, inversion requires a minimum dust temperature. Below $\sim 50 \mathrm{~K}$, the Planck function at $53 \mu \mathrm{m}$ decreases very rapidly with dust temperature. The flux can be increased by raising the dust opacity but cannot exceed the black-body limit, which is insufficient for inversion at dust temperatures below about $45 \mathrm{~K}$.

An important correlation is the increase of $L_{\mathrm{OH}}$ with $L_{\mathrm{IR}}$. Early OHMM observations revealed an approximately quadratic relation between the two (Baan 1989), but subsequent studies established the flatter dependence $L_{\mathrm{OH}} \propto L_{\mathrm{IR}}{ }^{1.2}$ (Darling \& Giovanelli 2002). Low gain amplification of background radio continuum implies that $L_{\mathrm{OH}}$ is proportional to the radio luminosity, which in turn is proportional to $L_{\mathrm{IR}}$ over the relevant luminosity range. Since the observed correlation is steeper than linear, Baan (1989) conjectured that maser optical depth $\tau_{\text {maser }}$ also varies linearly with the pump luminosity $L_{\mathrm{IR}}$. However, the Lockett \& Eitzur model results show that, depending on the different parameters, $\tau_{\text {maser }}$ in fact can either increase or decrease with the IR luminosity. The increase of $L_{\mathrm{OH}}$ in excess of linear proportion to $L_{\mathrm{IR}}$ may be explained by the results of the Gao \& Solomon (2004) of HCN emission from luminous IR galaxies. They find a tight proportionality between IR and HCN luminosities, and conclude that the amount of dense molecular gas, which is traced by the HCN, is linearly proportional to $L_{\mathrm{IR}}$. It is thus reasonable to assume that, together with the increase of dense molecular gas, the number of $\mathrm{OH}$ maser clouds is also increasing with $L_{\mathrm{IR}}$.

Line overlap is a major component of the OHMM inversion and the calculations show that the linewidth, $\Delta v$, is the most important factor in determining the $1667 / 1665 \mathrm{MHz}$ 
line ratio. Widths in excess of $\sim 2 \mathrm{~km} \mathrm{~s}^{-1}$ cause the $1667 / 1665$ ratio to be consistently larger than one, while widths $\lesssim 2 \mathrm{~km} \mathrm{~s}^{-1}$ can cause this ratio to be greater than or less than one, depending on the actual $\Delta v$. Large linewidths $\left(\gtrsim 10 \mathrm{~km} \mathrm{~s}^{-1}\right)$ produce ground state hyperfine transitions that are all inverted with approximately the same (negative) excitation temperature. Detailed comparison with observations is difficult because the satellite lines are very weak and have only been detected for relatively nearby sources. Even in those cases the signal to noise ratio is small, resulting in large uncertainties in line strength ratios. Figure 3 plots the $1667 / 1720 \mathrm{MHz}$ line ratio versus the $1667 / 1665$ $\mathrm{MHz}$ line ratio for four relatively nearby OHMM. The agreement is surprisingly good considering the large uncertainties in the line ratios.

The variation of line ratios with $\Delta v$ suggests that FIR might be a common pump of all $\mathrm{OH}$ main line masers, Galactic as well as megamasers, with the magnitude of velocity controlling line overlap the factor explaining the observed differences. The maser patterns in Galactic star forming regions stand apart because of the relatively small values of $\Delta v$ in these sources. The similarity between OHMM and evolved stars may reflect the large velocities controlling line overlap in both environments.

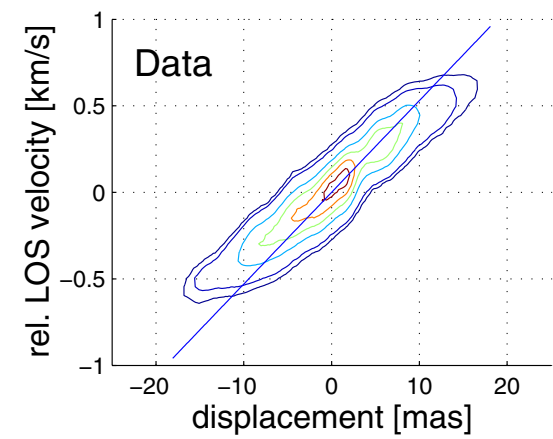

Figure 4. Position-velocity diagram of $12 \mathrm{GHz}$ methanol maser amplification contours in NGC 7538 IRS 1N (Pestalozzi et al. 2004).

\section{Disks and outflows}

Class II methanol maser emission often shows linear structures in both spatial maps and in position-velocity $(p-v)$ diagrams. It has been suggested that these structures delineate protostellar edge-on disks (Norris et al. 1998). However, similar structures can be produced also in bi-directional outflows, a geometry that actually seems more appropriate in a large fraction of methanol masers (De Buizer 2003). The fact that such disparate maser geometries can produce such similar signatures raises a fundamental question: Short of proper motion measurements, is there a reliable way to distinguish between the two? The methanol maser source towards IRS 1N in NGC 7538 may offer an answer. Its unique emission contours display a centrally-peaked large, elongated structure in the $p-v$ diagram, shown in figure 4 ; in contrast, most other sources typically display in such diagrams a string of maser features. The structure in NGC 7538 was successfully modeled in great detail by Pestalozzi et al. (2004) with an edge-on Keplerian disk. Although this interpretation was questioned by De Buizer \& Minier (2005), Kraus et al. (2006) have demonstrated the existence of jet precession in this source, settling the issue in favor of the disk geometry.

It turns out that the $p-v$ structure seen in figure 4 implies fairly tight constraints that significantly narrow the range of possible geometries (Pestalozzi et al. 2007). Consider first a bipolar outflow. When the outflow axis is in the plane of the sky, the emission 

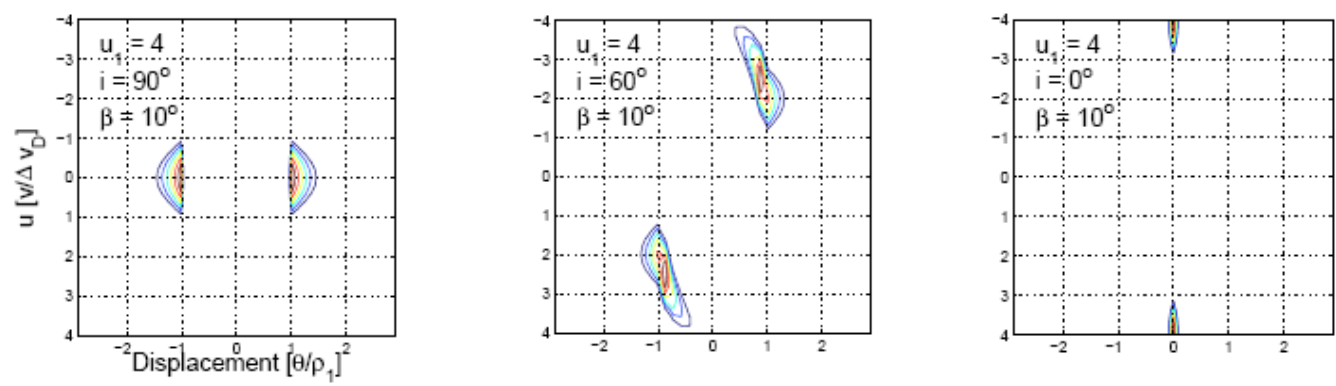

Figure 5. Position-velocity diagram of maser amplification contours for bipolar outflow with representative properties. The outflow axis is in the plane of the sky in the left panel, aligned with the line-of-sight in the right panel and $60^{\circ}$ off the line-of-sight in the center one (Pestalozzi et al. 2007).
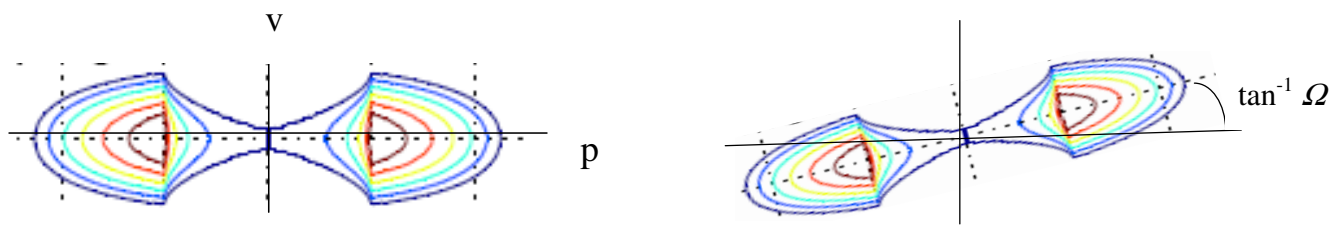

Figure 6. Position-velocity diagram for maser amplification in an edge-on disk. Left: A quiescent disk, only thermal velocities. Right: The same disk subjected to solid-body rotation with angular velocity $\Omega$ (Pestalozzi et al. 2007).

from each lobe is centered on velocity $v=0$. Because the density declines rapidly with radial distance, the emission is peaking at two regions displaced symmetrically from the outflow origin. The resulting structure in the $p-v$ diagram is two blobs that straddle the origin at $v=0$, as seen in the left panel of figure 5 . When the outflow axis is along the line-of-sight, the emission from both cones occurs at the same position, but at different velocities - blue and red shifted by the same amount from the systemic velocity. The $p-v$ diagram, shown in the right panel of figure 5 , again displays two blobs, only this time they are in the same position but at different velocities. In essence, rotating the outflow axis by $90^{\circ}$ effects a similar rotation of the emission contours in the $p-v$ diagram. It is not surprising that an intermediate orientation of the outflow axis likewise produces an intermediate rotation in the $p-v$ diagram, as can be seen from the center panel of figure 5 . Whatever the inclination of its axis, a bipolar outflow cannot produce the single centrally peaked structure seen in figure 4 . A smooth outflow will produce two mirror features displaced symmetrically from the center. Fragmentation of the outflow may introduce an asymmetry between the two halves but will always avoid the center, never producing a centrally peaked feature.

Consider now the case of an edge-on disk. When the disk does not rotate, its optical depth obeys $\tau(p, v)=\tau(p) \phi(v)$; that is, the positional variation of the amplification is controlled solely by the length of the amplifying column because the velocity profile $\phi$ is the same at every position. For uniform inversion, $\tau(p)$ is simply proportional to the pathlength through the disk, which is maximal at the two tangents to the disk inner radius. The $p-v$ diagram of the amplification contours in this case is shown in the left panel of figure 6. Similar to the outflow geometry, the contours show two distinct peaks, displaced symmetrically from the center. The center does show amplification in this case, but it is a local minimum. When the disk is set in rotation, the Doppler velocity of the emission from a point at radius $r$ and impact parameter $p$ obeys $v=\Omega(r) p$, where $\Omega(r)$ 
is the local angular velocity. Therefore, in solid body rotation the material along each impact parameter remains fully velocity coherent, only the velocity profile is centered on $v=\Omega p$; that is, the optical depth obeys $\tau(p, v)=\tau(p) \phi(v-\Omega p)$. As a result, the structure of amplification contours in the $p-v$ diagram remains the same, only rotated by the angle $\tan ^{-1} \Omega$ (and slightly stretched to maintain the peak positions at the two tangents to the inner radius), as shown in the right panel of figure 6. Similar to bipolar outflow, an edge-on disk rotating as a solid body will never produce a centrally peaked structure as observed in NGC 7538 (figure 4). Only differential rotation, such as Keplerian motion, can produce such structure. Furthermore, even in that case, turning the local minimum at the center of the $p-v$ diagram into a local maximum requires rotation rates in excess of a certain threshold (Pestalozzi et al. 2007). Detailed analysis of the $p-v$ diagram is, evidently, a powerful tool that deserves more study.

\section{Open problem - maser polarization}

Maser polarization behaves differently according to whether the Zeeman shift $\nu_{B}$ is larger or smaller than the linewidth $\Delta \nu_{D}$. The case $\nu_{B} \gg \Delta \nu_{D}$ is well understood. Spontaneous decays occur in pure $\Delta m$ transitions, producing in each transition radiation that is fully polarized and centered on a different Zeeman frequency. Under these circumstances the amplification process preserves the polarization, therefore thermal and maser polarizations are the same. The only difference between the two cases is the disparity between the $\pi$ and $\sigma$ maser intensities, reflecting their different growth rates (Elitzur 1996).

In the opposite limit, $\nu_{B} \gg \Delta \nu_{D}$, the Zeeman components overlap and the amplification mixes the different polarizations. The pumping processes produce radiation in three different modes of polarization with respect to the magnetic field $(\Delta m=0, \pm 1)$, but only two independent combinations propagate in any direction because the electric field must be perpendicular to the wave vector. Elimination of the longitudinal component implies specific phase relations among the three pump-generated electric fields; only waves launched with these phase relations produce superpositions that are purely transverse so that they can be amplified by propagation in the inverted medium. The phase relations translate to the following polarization for maser radiation propagating at angle $\theta$ from the magnetic axis:

$$
\frac{Q}{I}=-1+\frac{2}{3 \sin ^{2} \theta}, \quad \frac{V}{I}=\frac{16 x x_{B}}{3 \cos \theta},
$$

where $x=\left(\nu-\nu_{0}\right) / \Delta \nu_{D}$ and $x_{B}=\nu_{B} / \Delta \nu_{D}$. This solution, which was first derived by Goldreich, Keeley \& Kwan (1973) in the limit $x_{B}=0$ and extended by Elitzur (1996) to finite $x_{B}<1$, is obtained also from the requirement that the four Stokes parameters produce fractional polarizations that remain unaffected by the amplification process.

How the unpolarized radiation produced in spontaneous decays evolves into this stationary polarization solution remains an open problem. Numerical calculations that follow the growth of the Stokes parameters do not reach the stationary solution for any reasonable amount of amplification, and this slow growth can be understood from simple analytic considerations. However, the Stokes parameters are intensities, quadratic in the electric fields of the waves, and in formulating their growth equations all information about the phases of the electric vectors is lost. Since the stationary solution reflects specific phase relations between the $\pi$ and $\sigma$ components, the formalism employed for calculating the growth of the Stokes parameters negates the very effect it seeks to describe. Eliminating the phase information, the interactions selecting the stationary polarization 
are removed from the calculation. What is still missing is a full simulation of the ensemble evolution of interacting particles and electric fields. Such simulations are preformed in studies of laboratory plasma but have not yet been attempted for astronomical maser radiation.

\section{Coupled Escape Probability (CEP)}

Pumping models require reliable calculations of the population distribution in multilevel systems. The radiative terms in the statistical rate equations require the local radiation field, which must be determined from a separate solution of the radiative transfer equation for each and every line. Because of the complexity and computational demands of exact solution methods, most codes bypass altogether the radiative transfer equations, employing instead the escape probability approximation. In this approach only the level populations are considered, calculated from rate equations that include photon escape factors which are meant to account approximately for the effects of radiative transfer. This approach amounts to an uncontrolled approximation without internal error estimates because it is founded on a plausibility assumption right from the start. Nevertheless, this inherent shortcoming is often tolerated because of the simplicity and usefulness of the escape probability approach.

Elitzur \& Asensio Ramos (2006) have recently developed a new exact method, the Coupled Escape Probability (CEP), that retains all the advantages of the naive escape probability approach. In this new technique the source is divided into zones, and formal level population equations are derived rigorously from first principles. Different zones are coupled through terms resembling standard escape probability expressions, resulting in a set of coupled algebraic equations for level populations that are self-consistent with the line radiation they generate. Any desired accuracy can be achieved by increasing the number of zones. In comparative studies of a number of standard problems, the CEP method outperformed the current leading techniques by substantial margins. While the new method holds a great performance edge, its greatest advantage is its simplicity and ease of implementation. The CEP method employs a set of algebraic equations that are already incorporated in all codes based on the escape probability approximation. All that is required for an exact solution is to augment the escape probability in such codes with a zone-coupling sum. With this simple modification, the multi-level line transfer problem is solved exactly and efficiently.

For my own pumping calculations I developed over the years the escape probability code MOLPOP that can handle any arbitrary atom or molecule for which atomic data exist. For any species, the energy levels, collision rates and Einstein A-coefficients are tabulated in ordinary ASCII text files and MOLPOP selects from its database the appropriate files according to input instructions. We are in the process of implementing the CEP technique into MOLPOP, creating a general purpose tool for atomic and molecular line analysis that will become publicly available. In addition, the atomic and molecular data team at Meudon Observatory has embarked on the development of another tool that accesses through the web their central database and creates on the user's local computer atomic and molecular data files in the format required by MOLPOP. This auxiliary tool will become part of the MOLPOP/CEP package and will provide users the most current atomic data at all times. The complete package will enable exact pump modeling for every maser molecule. We hope to have a first release by the end of 2007 .

\section{Acknowledgements}

The author's maser research is supported by NSF through grant AST-0507421. 


\section{References}

Baan, W. 1985, Nature, 315, 26

Baan, W. 1989, ApJ, 388, 804

Baan, W. A., Haschick, A., \& Henkel, C. 1989, ApJ, 346, 680

Baan, W. A., Haschick, A., \& Henkel, C. 1992, AJ, 103, 728

Darling, J. 2003, Physical Review Letters, 91, 011301

Darling, J. \& Giovanelli, R. 2002, AJ, 124,100

De Buizer, J. 2003, MNRAS, 341, 277

De Buizer, J. M., \& Minier, V. 2005, ApJ, 628, L151

Elitzur, M. 1992, Astronomical Masers (Kluwer Academic Publishers)

Elitzur, M. 1996, ApJ 457, 415

Elitzur, M. \& Asensio Ramos, A. 2006, MNRAS 365, 779

Gao, Y., \& Solomon, P. M. 2004, ApJ, 606, 271

Goldreich, P., Keeley, D. A. \& Kwan, J. Y. 1973, ApJ, 179111

Henkel, C., \& Wilson, T. L. 1990, A\&A, 229, 431

Kanekar, N., et al. 2005, Physical Review Letters, 95, 261301

Kraus, S., et al. 2006, A\&A, 455, 521

Lockett, P. \& Elitzur, M. 2007, in preparation

Lonsdale, C. J. 2002, Cosmic Masers: From Proto-Stars to Black Holes, IAU Symposium \#206, 413

Martin, J., Bottinelli, Dennedeld, M., Gougenheim, L., \& Lesqueren, A., 1989, A\&A, 208,39

Migenes, V., \& Reid, M. J. 2002, Cosmic Masers: From Proto-Stars to Black Holes, IAU Symposium \#206 (San Francisco: ASP)

Momjian, E., Romney, J. D., Carilli, C. L., \& Troland, T. H. 2006, ApJ, 653, 1172

Murphy, M. T., Webb, J. K., Flambaum, V. V., Dzuba, V. A., Churchill, C. W., Prochaska, J. X., Barrow, J. D., \& Wolfe, A. M. 2001, MNRAS, 327, 1208

Parra, R., Conway, J.E., Elitzur, M., \& Pihlstrom. Y.M. 2005, A\&A, 443, 383

Pestalozzi, M. R., Elitzur, M., Conway, J. E., \& Booth, R. S. 2004, ApJ, 603, L113; Erratum: 2004, ApJ, 606, L173

Pestalozzi, M. R., Elitzur, M. \& Conway, J. E., 2007, in preparation

Pihlström, Y. M., Conway, J. E., Booth, R. S., Diamond, P. J., \& Polatidis, A. G. 2001, A\&A, 377, 413

Norris, R., Byleveld, S., \& Diamond, P. J. 1998, ApJ, 508, 275

Rieu, N. Q., Winnberg, A., Guibert, J., Lepine, J. R. D., Johansson, L. E. B. \& Goss, W. M. 1976, A\&A, 46, 413

Weisberg, J. M., Johnston, S., Koribalski, B. \& Stanimirovic, S. 2005, Science 309, 106 